\title{
Pomegranate (Punica granatum L.) Processing, Value Addition and their Medicinal Properties Related to Human Health: A Review
}

\author{
M. Viswanath ${ }^{1}$, P. Sridevi ${ }^{1}$, K. Venkataramudu ${ }^{1}$, S.M. Rajesh Naik ${ }^{1^{*}}$ \\ and K. Ravindra Kumar ${ }^{2}$ \\ ${ }^{1}$ Department of Fruit Science, ${ }^{2}$ Department of Floriculture and Landscaping, Horticultural \\ College and Research Institute, Dr. Y.S.R. Horticultural University, \\ Venkataramannagudem - 534101, Andhra Pradesh, India
}

*Corresponding author

\section{A B S T R A C T}

\begin{tabular}{|l|}
\hline K e y w o r d s \\
$\begin{array}{l}\text { Pomegranate, } \\
\text { Antioxidants, Free } \\
\text { radicals, Ellagic } \\
\text { acid, Value addition }\end{array}$ \\
\hline Article Info \\
\hline $\begin{array}{l}\text { Accepted: } \\
\text { 12 December } 2018 \\
\text { Available Online: } \\
\text { 10 January } 2019\end{array}$ \\
\hline
\end{tabular}

Pomegranate (Punica granatum L.) is a deciduous shrub, native to Iran. Now-a-days, besides its use as a fruit, its medicinal properties have attracted the interest of researchers of many countries. Both in vitro and in vivo studies have demonstrated how this fruit acts as antioxidant, antidiabetic, hypolipidemic, antiviral, and anticarcinogenic activities. The fruit also improves cardiovascular and oral health. These health benefits of pomegranate have been attributed to its wide range of phytochemicals and the ellagic acid is one of the main components of pomegranate with phenolic structure and antioxidant activity which are predominantly impact on human health. However, pomegranate fruits are prone to postharvest water loss, chilling injuries, physical disorders and fungal diseases. The aim of this review was to present an overview of the functional and medical properties of this fruit and to present the recent advancements, current status and developments in the pomegranate processing and their utilization. It also focuses on the increasing interest in the search for new pomegranate derived food products, the application of the new post harvest technologies and identifies future prospects for the development and utilization of pomegranate processing.

\section{Introduction}

The pomegranate (Punica granatum L.) is an ancient fruit; it has been widely consumed in various cultures for thousands of years. The use of pomegranate fruit dates back to Biblical times and reports of its therapeutic qualities have echoed throughout the millennia (Longtin, 2003). In Chinese the seeds symbolized longevity and immortality. Science from the ancient times pomegranate has been regarded as a "healing food" with numerous beneficial effects in several diseases (Vidal et al., 2003). Indeed, the pomegranate was commonly used in folk medicine, for eliminating parasites, as an antihelmintic and vermifuge, and to treat and cure ulcers, diarrhea, acidosis, dysentery, hemorrhage, microbial infections, and respiratory pathologies. It was also used as an antipyretic (Larrosa et al., 2010). Recent years have seen increased interest on the part 
of consumers, researchers, and the food industry into how food products can help maintain health; and the role that diet plays in the prevention and treatment of many illnesses has become widely accepted (ViudaMartos, 2010). At the present time, considerable importance is given to functional foods, apart from their basic nutritional functions, provide physiological benefits and play an important role in disease prevention or slow the progress of chronic diseases (Viuda-Martos, 2010). Pomegranate is known as a super fruit of next generation, owing to its low maintenance cost, tolerant to biotic and abiotic stresses, excellent flavour, nutritive value and medicinal properties.

In India, in spite of the known nutraceutical benefits and great global demand for potentially pomegranate derived products; the pomegranate processing industry is not developed due to lack of technological developments for commercialization, resource personnel and scientific research database. Thus the post harvest losses are very high (20-40\%). About 10- 15\% fresh produce lose their market value and consumer acceptability due to improper post harvest management. Minimizing these losses can increase their supply without bringing additional land under cultivation. A number of processed products can be manufactured and preserved for future time satisfying the consumer perception of a high nutritional quality and convenience produce. These could also help to have the good returns and make the availability of the fruit throughout the year increasing the shelf life of the pomegranate fruits as such for a considerable period. Potentiality for processing into value added products having extended shelf life, yielding potential, better keeping quality and higher nutraceutical value, popularity of pomegranate is increasing among the growers and consumers worldwide.

\section{The metabolites of Punica granatum}

About $50 \%$ of the total fruit weight corresponds to the peel, which is an important source of bioactive compounds such as phenolics, flavonoids, ellagitannins (ETs), and proanthocyanidin compounds. The edible part of the pomegranate fruit $(50 \%)$ consists of $40 \%$ arils and $10 \%$ seeds. Arils contain $85 \%$ water, $10 \%$ total sugars, mainly fructose and glucose, and $1.5 \%$ pectin, organic acid such as ascorbicacid, citricacid, malicacid, and bioactive compounds. The seeds are a rich source of total lipids; pomegranate seed oil comprises $12 \%$ to $20 \%$ of total seed weight. The oil is characterized by a high content of polyunsaturated (n-3) fatty acids such as linolenic, linoleic, and other lipids such as punicic acid, oleic acid, stearic acid, and palmitic acid. The seeds also contain protein, crude fibers, vitamins, minerals, pectin, sugars, polyphenols, isoflavones (mainly genistein), the phytoestrogen coumestrol, and the sex steroid, estrone.

\section{Application of pomegranate in traditional medicine}

All components of pomegranate fruit with abundant tannins show relatively strong astringent effects. Several infusions or decoctions of the plant flowers have been used in traditional medicine to treat simple diarrhea, vaginal discharge, and also this extract accompanied with pomegranate peel have usually been gurgled to relieve pancreas inflammation of the pancreas. Refreshing juice of the fruit is recommended to heal gallbladder diseases. Its decoction appears to be helpful for treating diseases such as ordinary diarrhea, dysentery, and stomach disorders. Tannin content of pomegranate seed, however, is not remarkable and it is usually used to treat women vaginal discharge and wound healing. Fresh or dried root barks or ethanol extracts of pomegranate are used to 
remove intestinal parasites due to the alkaloid substances. It is also used in traditional medicine because of the antibacterial and anti-inflammatory properties.

\section{Pharmacological pomegranate}

\section{properties}

of

Flavonoids and tannins of pomegranate juice can prevent the growth of cancer cells. Flavonoids observed in the watery extract and fruit peel have shown estrogenic activity. In addition, luteolin and naringenin have indicated an activity similar to the hormone usually secreted prior to pregnancy in women. Polyphenols of fermented extract of pomegranate fruit potentially appear to have antioxidant activity and pericarp tannins may increases antioxidant potential of fruit extract. The stronger activities in polyphenols of fermented extract than nonfermented extract is likely due to the breakdown of flavonoids sugar complexes during fermentation that the final products will contain high concentrations of free polyphenols (with high biological activity).

Ellagic acid and gallic acid are among the constituents observed in pomegranate peels, and the former is a dimeric derivative of gallic acid and is found mostly in higher plants, such as fruits and nuts. Ellagic acid shows antimutant, antiviral, antioxidant, and skin-bleaching activity and has already been added to food as an antioxidant in Japan. Antioxidant capacity of extracts derived from pomegranate peel in producing phospholipid complex has been measured. Antioxidant capacity of extracts from pomegranate peels is due to the presence of the phenols such as ellagic tannins, ellagic acid, and gallic acid. Antimutagenic and anticarcinogenic properties of the extracts were examined against the azide sodium by the Ames test. The experiment showed that juice extract of pomegranate peel can inhibit mutation and cancer using azide sodium in 2 species of salmonella.

More recent studies have shown that the pomegranate fruit are enriched with a strong antioxidant called punicalagin that controls superoxide and free radicals of DPPH $(1,1-$ diphenyl-2-picrylhydrazyl). The best extraction and separation way is done by methanol. Punicalagin is capable to regulate the activity of superoxide and DPPH radicals. In an additional research, the blood plasma derived from person who had taken pomegranate juice containing ellagic acid (25 $\mathrm{mg}$ ) and ellagitannins (318 mg, especially punicalagin) was analyzed. The main purpose of the earlier study was to assess the amount and time duration of ellagic acid bioavailability in plasma following consumption. According to the results, the highest ratio of ellagic acid in the blood plasma was measured at $0.5,1,2,3,4,5$, and 6 hours after taking pomegranate juice. The highest and lowest ratios were observed at 1 hour and 4, 5, and 6 hours after consumption, respectively. The presence of the free ellagic acid in the blood plasma is induced by its breakdown under biologic $\mathrm{pH}$ of stomach. Thus, it can be used as a biologic marker in bioavailability studies confirming the consumption of ellagic acid from food resources. Polyphenols of oil prohibit the activity of eicosanoid and cyclooxygenase enzymes. 18C Trans fatty acids known as conjugated linoleic acid, structurally related to punicic acid, possess cancer arresting properties.

Punic acid acts as an inhibitor of prostaglandin biosynthesis as well as a cytotoxin for cancer w2a cells; such activity is possibly due to the inhibitory effect against fat peroxidation. Punic acid of pomegranate seed oil inhibits prostaglandin biosynthesis (promote ornithine decarboxylase enzyme activity at lower concentration). Also this oil 
can prevent DMBA- and TPA-induced skin cancer. Inhibitory activities of prostaglandin as well as antioxidant activity of polyphenols extracted from both pomegranate seed oil and its fermented extract have widely been reported for prevention from human breast cancer. Inhibitory impact of the watery and oily parts of the fruit has been reported on breast cancer cells in vivo. Such parts prohibit the activity of enzymes responsible for active estrogen biosynthesis (17-b-estradiol). Since the watery and oily parts of the fruit are chemically different, they probably active different mechanisms in the prevention of cancer. Pomegranate seed oil is considered as biosynthesis inhibitor E2 (17-b-estradiol) catalyzed by 17-b-hydroxysteroid enzyme. It also prevents invasion of cancer cells and also can strengthen and encourage apoptosis. Extracted polyphenols of pomegranate seed oil can potentially prevent cyclooxygenase activity. Inactivation of the mentioned enzyme prevents the proliferation of breast cancer cells.

Despite these high beneficial compounds towards human health, a number of processed products manufactured and preserved for future time satisfying the consumer perception of a high nutritional quality and convenience produce. Potentiality for processing into value added products having extended shelf life, yielding potential, better keeping quality and higher nutraceutical value, popularity of pomegranate is increasing among the growers and consumers throughout the world.

\section{New technologies for pomegranate storage}

The pomegranate is classified as a nonclimacteric fruit. In spite of the non climacteric nature of the fruit, quantitative and qualitative loss still occur due to postharvest handling processes, resulting in chilling injuries, husk scalding, weight loss and decay of pomegranate. The new physical treatment applications have been reported to prolong the shelf life of the fresh pomegranates. Kader et al., (1984) and Artes (1992) recommended a fast pre-refrigeration using forced air as one of the simplest ways to extend the commercial life of pomegranate up to 2-3 months by keeping storage temperature around $5^{\circ} \mathrm{C}$. Active MAP involves a quick process of gas flushing or gas replacement or the use of gas-scavenging agents to establish a desired gas mixture within the package. Studies have shown that modified atmosphere packaging (MAP) and controlled atmosphere storage (CAS) have the ability to delay quality loses and thus extends the shelf life of fresh or minimally processed or fresh-cut produce.

\section{MAP sensing and monitoring}

'Smart' or 'active' or 'intelligent' packaging system is introduced to improve the safety of MAP products and to extend the technology to a broader spectrum of products. The Smart packaging as an interaction between the packing system and the product itself which confers intelligence appropriate to function and use of the product with the ability to sense or to be sensed and to communicate. Nano biosensors can serve as the best smart packaging tool for MAP sensing and monitoring. Artes et al., (1998) recommended a controlled atmosphere of $5 \% \mathrm{O}+0 \%$ to $5 \%$ $\mathrm{CO}$ composition for the storage of Mollar pomegranate at $5^{\circ} \mathrm{C}$ with $\mathrm{RH} 95 \%$ to minimize decay weight loss and chilling injuries.

In contrast, (Kader, 1995) recommended a gas composition of $3 \%$ to $5 \% \mathrm{O}+5 \%$ to $10 \% \mathrm{CO}$ for storage of pomegranate at $5^{\circ} \mathrm{C}$. Studied also reported the chilling injuries to pomegranates when stored at temperatures lower than $5^{\circ} \mathrm{C}$. 


\section{Pomegranate juice processing}

Pomegranate contains 48 to 52 per cent of edible part on the whole fruit basis, which comprises of 78 percent juice and 22 percent seed. The seeds along with arils are crushed and juice is extracted and marketed as a fresh juice due to its excellent flavour, attractive fragrance, delicious taste and high nutritive and medicinal value. Production of juice from the pomegranate arils proved to be one of the important methods of value addition. The juice can be processed possible into the squash, syrup, nectar, jelly, concentrate and such other products. Pomegranate juice can be used as an ingredient providing colour to the other products. The pomegranate juice is a rich source of polyphenols. The phenolic constituents of pomegranate such as the anthocyanins give the colour and other polyphenols such as flavonoids and some non-flavonoids are responsible of antioxidant properties, astringency and bitterness to juice. The antioxidant qualities of pomegranate juice, makes it appealing for the production of health supplements and nutraceuticals.

\section{Clarification of pomegranate juice}

Clarification or fining is one of the most important steps in fruit juice processing. The procedure helps to remove active haze precursors and thus decrease the potential for haze formation during storage. The rind of the pomegranate fruit contains a very large amount of excessively 'puckery' tannin which enters the juice and makes it undrinkable if the whole fruit is crushed or pressed with excessive pressure. Nutritionist, however, recommend in contrast to preserve these compounds during the fruit juice processing because of their health protective effects. Pomegranate juice contains only trace amount of pectin. Therefore it can be filtered easily after pressing without clarification. However clarification is necessary to prevent the formation of cloudy appearance during storage and also to improve the taste of the product. If the clarification is not employed, the product has bitter taste due to high tannin content. For clarification, gelatin, bentonite, clays etc. may be used as flocculating agent. The centrifugation method may also be employed for the clarification of the fruit juices. The most effective method to remove the phenolic compounds in pomegranate juice was the conventional fining with gelatin (300 $\mathrm{mg} / \mathrm{l})$ and bentonite $(300 \mathrm{mg} / \mathrm{l})$ along with poly-vinyl-poly-pyrrolidone (PVPP). The use of pectinase enzyme for the clarification of the fruit juices by depectination is reported by many authors.

\section{Packaging of pomegranate juice}

Packaging material selection as well as processing influences the quality of foods, altering colour and nutrient composition during storage as a result of contact with oxygen and light transmission through them. Paperboard cartons with low density polyethylene (LDPE) coating or glass containers are commonly used materials of juices. Oxygen and light have destructive effect on the anthocyanin during storage. So the packaging material also plays an important role in the colour stability of stored pomegranate product. Perez- Vicente et al., (2004) assessed the influence of packaging material on colour and bioactive compounds of pasteurized pomegranate juice during storage at $24 / 18{ }^{\circ} \mathrm{C}$ and $40-50 \%$ RH. Glass containers were found to be better as compared to high density polyethylene or polyvinylchloride containers with regards to retention of anthocyanins, vitamin $\mathrm{C}$ and organoleptic quality of the fruit juices.

\section{Storage of pomegranate juice}

The numbers of factors are affecting the stability of the coloured pigments like 
anthocyanin in the pomegranate juice includes temperature, oxygen, light, $\mathrm{pH}$, enzymatic action etc. Out of these, the storage temperature is the important one. Many researchers have reported that the pomegranate juice can be successfully stored at ambient temperature as well as in cold storage $\left(5 \pm 12^{\circ} \mathrm{C}\right)$. But the better retention of anthocyanins and reduction in the enzymatic activity of pomegranate juice was reported in the pomegranate juice stored in cold storage.

\section{Fresh pomegranate arils}

Ready to eat pomegranate arils has been a challenge that has been approached by several research groups in Spain and the USA. In recent years, minimally processed "ready-toeat" pomegranate arils have become popular due to their convenience, high value, unique sensory characteristics and health benefits. James Caleb et al., (2011) reported that with increasing global interest in postharvest handling and nutritional value of pomegranate. Gil et al., (1995) reported that the pomegranate arils of Mollar variety washed with chlorinated water $(100 \mathrm{mg} / \mathrm{kg})$ and antioxidants solution $(5 \mathrm{~g} / \mathrm{l}$ ascorbic acid and $5 \mathrm{~g} / \mathrm{l}$ citric acid), packed in OPP film, using an initial atmosphere actively modified to $0 \mathrm{ml} / \mathrm{l} \mathrm{CO}$ and $20 \mathrm{ml} / 1$ and stored at $1{ }^{\circ} \mathrm{C}$, can be stored up to 7 days maintaining a good quality and appearance, without visible attack of moulds or off flavour developments. Sepulveda et al., (2000) investigated that pomegranate arils cv. Espanola washed and immersed in antioxidant solution (sodium hypochlorite, $200 \mathrm{ppm}, 5 \%$ ascorbic acid and $5 \%$ citric acid) for 1 minute and stored at $5 \pm$ $0.5^{\circ} \mathrm{C}$ in three semi-permeable packaging can be stored for 7 days with commercial marketable acceptance.

\section{Frozen arils}

After preparing the arils same as that of minimally processed arils, they were packed in the polyethylene bags with syrup of $15^{\circ}$ Brix. This concentration is similar to that of the arils. The arils were then frozen in a chest freezer. The juice contain of aril enters the syrup during freezing. Maestre et al., (2000) also reported the freezing of the arils coated with the sugar. The coated sugar turned red during freezing and storage. The arils should be eaten frozen to avoid an excessive loss of turgence.

\section{Appertised arils}

Appertised arils are the product prepared by putting the arils in syrup of $15^{\circ}$ Brix and packed into metal tin. The tins were heated, sealed and sterilized for 10 minutes. After stabilization, most of the tins had arils that were too soft and that tasted cooked. The tins which were prone to less severe heat treatment had adequate textured arils with good taste.

\section{Arils in vinegar}

The arils were preserved in vinegar with an acidity of 5\% and packed in jar. The resultant product was the brown coloured pomegranate arils.

\section{Jellies}

An attractive jelly can be prepared from pomegranate juice. Adsule et al., (1995) prepared good pomegranate jelly on a small scale from Ganesh cultivar of pomegranate. While making jellies, approximately $50 \%$ of the anthocyanins present in pomegranate juice were lost. Maestre et al., (2000) investigated that the acidification of juice produced a noteworthy improvement in the colour of jelly, both initially and during storage. During storage, certain colour differences were observed, which indicates that the $\mathrm{pH}$ was not only the parameter responsible for this characteristic. 


\section{Pomegranate molasses}

It is traditional Middle Eastern ingredient made from cooked down pomegranate juice. Thick and syrupy in texture, pomegranate molasses provide tangy flavour and is dark in colour. Its sweetness comes from the concentration of the fruits natural sugars. To make $1 / 2$ cup of molasses, 4 cups of juice is heated in a pan for 45 minutes, allowing it to thicken but not overcooked. The product can be stored in air tight container under refrigerated conditions for 3 months. It is typically used to flavour chutneys, curries and salad dressings to glaze or tenderize meat products.

\section{Pomegranate syrup/grenadine}

Pomegranate syrup is sold commercially as grenadine and is used as flavouring in alcoholic drinks, soft drinks and confections. Grenadine is light pomegranate syrup prepared by mixing juice and sugar. A syrup of about $60^{\circ}$ Brix with an added acidity of $1.5 \%$ as citric acid has a bright purplish red colour and a delightful taste and flavour. It can be preserved by pasteurization or by addition of sodium benzoate.

\section{Wine}

Pomegranate juice can be utilized to make good quality wine. Sugar is added to adjust Brix to $22-23^{\circ}$. The pasteurized juice is fermented with starter wine yeast. The fermentation is allowed to continue until desired level of alcohol is obtained. The wine is clarified by bentonite treatment or by centrifugation. The wine aged in the same manner as red grape wine.

\begin{tabular}{|l|l|}
\hline \multicolumn{1}{|c|}{ Plant component } & \multicolumn{1}{c|}{ Constituents } \\
\hline Pomegranate juice & $\begin{array}{l}\text { Anthocyanins, glucose, organicacid, ascorbic acid, EA, ETs, } \\
\text { gallic acid, caffeicacid, catechin, quercetin, rutin, minerals. }\end{array}$ \\
\hline Pomegranate seed oil & $\begin{array}{l}\text { Conjugated linolenic acid, linoleicacid, oleicacid, stearic } \\
\text { acid, eleostearic acid, catalpic acid }\end{array}$ \\
\hline Pomegranate peel & $\begin{array}{l}\text { Luteolin, quercetin, kaempferol, gallagic, EA glycosides, EA, } \\
\text { punicalagin, punicalin, pedunculagin }\end{array}$ \\
\hline Pomegranate leaves & EA; fattyacids \\
\hline Pomegranate flower & Polyphenols, punicalagin punicalin, EA \\
\hline Pomegranate rootsandbark & Alkaloids,ETs \\
\hline
\end{tabular}

\section{Pomegranate waste utilization}

All parts of the pomegranate tree i.e. roots; bark, leaves, flowers, rind and seeds can be processed for value added products having medicinal, industrial and cosmetic value. Technology for preparation of rind powder has been developed, having potential uses in medicine, leather and dye industry and in tooth powder preparation. The recovery of rind powder has been found to be $15.5 \%$ on whole fruit basis and $34 \%$ on rind weight basis.
In conclusions the consumption of pomegranate has grown tremendously due to its reported health benefits. Pomegranate and derivatives, such as juice, peel, and seeds, are rich sources of several high-value compounds with potential beneficial physiological activities. The rich bioactive profile of pomegranate makes it a highly nutritious and desirable fruit crop. Accumulating research offers ample evidence that routine supplementation with pomegranate juice or extract may protect against and even improve several diseases, including diabetes and 
cardiovascular disease; it may even help to prevent and arrest the development of certain cancers, in addition to protecting the health of the mouth and skin. Side effects are very rare. Using concentrated, low-cost pomegranate juice or standardized pomegranate extract capsules offers consumers a way of reaping the broad spectrum of health benefits of this fruit. Last few years, the research and development activities on pomegranate fruit have aimed to develop technologies for new pomegranate derived food products. The pomegranate can be processed into products like minimally processed fresh arils, juice, squash, beverage, molasses, juice concentrates, frozen seeds, jam, jelly, marmalades, grenadine, wine, seeds in syrup, pomegranate spirits, pomegranate powder, pomegranate rind powder, anardana, confectionery, pomegranate seed oil etc., for the popularization of pomegranate based products.

\section{References}

Artes F, Gil MI, Martinez JA (1995) Procedimiento para la conservation de semillas de granada en fresco.

Artes, F, Marin, J.G, Martinez, J.A. (1998). Permeability rates of films for modified atmosphere packaging of respiring foods. Food quality Leuven: European Commission: 153-157.

Artes, F.(1992). Factores de calidad y conservacion frigorifica de la Granada. II Jornadas Nacionales del Granado. Universities Politecnica de Valencia Valencia.

Chavan, U.D, Adsule, R.N, Kadam, S.S. (1995). Physico-chemical properties of pomegranate rind powder. Beverage and FoodWorld. 22: 36.

James Caleb O, Opara UL, Witthuhn CR (2011) Modified atmosphere packaging of pomegranate fruit and arils: A review. Food Bioprocess
Technology Published Online by Springer Science Business Media LLC.

Kader, A.A, Chordas, A, Elyatem, S.(1984). Response of pomegranates to ethylene treatment and storage temperature. California Agriculture. July-August: 14-15.

Kader, A.A. (1995). Regulation of fruits physiology by controlled and modified atmosphere. Acta Horticultural. 398: 59-70.

Larrosa M, Gonz Alez-Sarrias A, Y, A NezGascon, M.J, Selma, M.V, AzorınOrtuno, M, Toti, S, Tomas-Barberan, F, Dolara, P, Espina, J.C. (2010). Anti-inflammatory properties of a pomegranate extract and its metabolite urolithin-A in a colitis rat model and the effect of colon inflammation on phenolic metabolism. J Nut Biochem. 21(8):717-25.

Maestre, J, Melgarejo, P, Tomas-Barberan, F.A, Garcia-Viguera, C. (2000). New food products derived from pomegranate. Symposium on production processing and marketing of pomegranate in the Mediterranean region. Advances in research and technology, Spain. 243-245.

Perez-Vicente, A, Serrano, P, Abellan, P, Garcia-Viguera, C. (2004). Influence of packaging material on pomegranate juice colour and bioactive compounds during storage. Journal of Science and Food Agriculture 84: 639-644.

Sepulveda, E, Galletti, L, Saenz, C, Tapia, M. (2000). Minimal processing of pomegranate var. Wonderful. Symposium on production processing and marketing of pomegranate in the Mediterranean region: advances in research and technology, Zaragosa Spain: 237-242.

Vidal, A, Fallarero, A, Pena, B.R, Medina, M.E, Gra, B, Rivera, F, Gutierrez, Y, 
Vuorela, P.M. (2003). Studies on the toxicity of Punica granatum L. (Punicaceae) whole fruit extracts. Journal of Ethnopharmacol. 89:295300.

Viuda-Martos, M, Ruiz-Navajas, Y, Fern an
dez-Lopez, J, Perez-Alvarez, J.A. (2010). Spices as functional foods: a review. Crit Rev Food Sci Nut In Press.

\section{How to cite this article:}

Viswanath, M., P. Sridevi, K. Venkataramudu, S.M. Rajesh Naik and Ravindra Kumar, K. 2019. Pomegranate (Punica granatum L.) Processing, Value Addition and their Medicinal Properties Related to Human Health: A Review. Int.J.Curr.Microbiol.App.Sci. 8(01): 17221730. doi: https://doi.org/10.20546/ijcmas.2019.801.183 